\title{
Octonians and the EM-Compass
}

\section{Gudrun Kalmbach HE*}

Mathematic Informatics Naturewissenchaften, Technic, Germany

*Corresponding Author: Gudrun Kalmbach HE, Mathematic Informatics

Naturewissenchaften, Technic, Germany.
Received: February 02, 2021

Published: February 26, 2021

(C) All rights are reserved by Gudrun

Kalmbach HE.

\section{Abstract}

In the first section, the EM compass is described and an application to leptons. Since the octonians are used, a Hilbert subspace lattice structure is described: why the extension from spacetime coordinates to octonians necessary.

Keywords: EM-Compass; Octonians

\section{EM-compass}

The EM-compass is the $8^{\text {th }}$ roots version of the G-compass which is the $6^{\text {th }}$ roots version of a dihedral compass. If octonians are generated by doubling the quaternions, the two leptonic series are listed with their EM properties as signed rotating EM (neutral) charge, magnetic momentum (momentum) and induction (oscillation) and the octonians are marked as $8^{\text {th }}$ roots of unity on the EMcompass circle with the eight properties added. In comparison, for the G-compass six color charges are added to the $6^{\text {th }}$ roots of unity (Figure 4). The matrix $G$ is of order 6 , the matrix $i^{1 / 2}$ is of order 8. It has as scalar $1 / \sqrt{2}$ and the first row of the matrix $B$ is $(1-1)$, the second row (1 1). For the G-compass the conjugation operator associated a color charge $\mathrm{x}=\mathrm{r}, \mathrm{g}$, $\mathrm{b}$ with its dual $\mathrm{c}(\mathrm{x})$ turquoise, magenta, yellow and the Heisenerg uncertainties did arise: $x, c(x)$ are drawn as two rays on a coordinate axis $u$ as directions $u$, $-u$ where $\mathrm{u}$ is the coordinate $\mathrm{x}, \varphi, \mathrm{t}$ and $\mathrm{u}$ is momentum, angular momentum, energy.

For the EM-compass the weak decay associates with $\mathrm{e}^{+}$(positron) the neutrino $v$, with e- the antineutrino $c(v)$, magnetic momentum $\mu$ the momentum $\mathrm{p}=\mathrm{mv}$ and with the EM induction the (anti-)neutrino oscillation. The last pair is for an octonian EM-

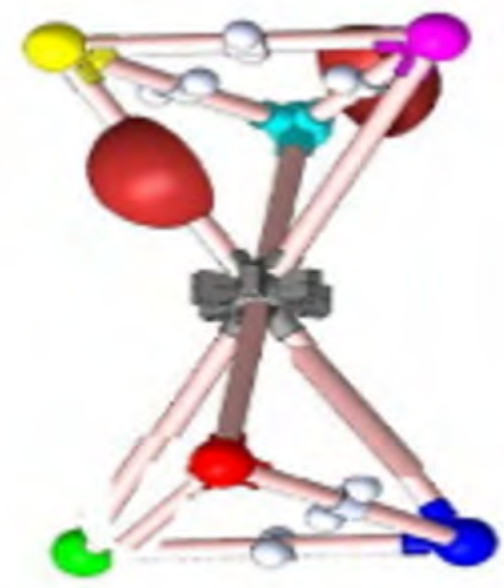

Figure 1: Deuteron with two nuceon tetrahedrons, red $r$ is the $\mathrm{x}$-axis, green the $\mathrm{y}$-axis, blue the $\mathrm{z}$-axis. 
compass: For the G-compass the octonian first $\mathrm{e}_{0}$ and last $\mathrm{e}_{7}$ coordinates have been used as a (eigen)vector setting units for measures or angles $\theta$ for two rays at a common origin with this angle in between and $e_{7}$ was rolled to a Kaluza-Klein circle for the complex polar exponential function $\exp (i \varphi)$ as circumference $U(1)$ of the compass. In this sense, induction is a cross product vector for $\mathrm{e}_{0}$ as rotational EM momentum, area differentiated from a magnetic flow (or reversely the flow integrated from induction). The U(1) circle $\left(e_{7}\right)$ is replaced by an oscillation as $g=\exp \left(i\left(\omega t+\varphi_{0}\right)\right)$,

t time, $\omega=d \varphi / d t$ angular speed, $\varphi_{0}$ a phase angle. The oscillation is interpreted as a pendulum motion for neutrinos (or antineutrinos) in figure 4. The former datas $(v, p, g)$ are weights of a Gleason frame GF orthogonal base triple with quaternionic coordinates, attaching Pauli matrices to the triple $(v, \mathrm{p}, \mathrm{g})$ and adding the identity matrix. The $p, p_{j}$ vector has three values for neutrinos three masses $m_{j}$. The oscillation $g$ is discrete, cyclic with the $B^{2}$ matrix powers as $1, \mathrm{i},-1,-\mathrm{i}$ and back to 1 . At rest, the observable neutrino is $\left(v, \mathrm{p}_{1}, \mathrm{~g}\right)$ with the smallest mass. In the first application of $g$, substituting $\varphi$ $=\pi / 2$ for $\left(\omega t+\varphi_{0}\right)$ for the turn of the compass needle, $1 \rightarrow \mathrm{i}$ a Higgs field for mass makes the triple $\left(v, p_{2}, g\right)$ observable, then in sequence $\mathrm{i} \rightarrow(-1)\left(v, \mathrm{p}_{3}, \mathrm{~g}\right)$ is observable, $(-1) \rightarrow(-\mathrm{i})\left(v, \mathrm{p}_{2}, \mathrm{~g}\right),(-\mathrm{i}) \rightarrow 1\left(v, \mathrm{p}_{1}, \mathrm{~g}\right)$. The Higgs field is replacing an added $r g b$-graviton to three quarks in a nucleon where a similar SI-rotor is postulated.

The oscillation corresponds to it. This is not named a WI-rotor since it applies for neutral leptons, but not for the electrical charged EM leptons. For them the Hopf fiber bundle makes another spherical, 2-dimensional model as rotating sphere $S^{2}$ (Figure 2). On the north pole $\infty$ of $S^{2}$ sit the spin plus attached magnetic momentum (replacing $\mathrm{p}$ ) vectors for the rotation axis towards the south pole 0 of $\mathrm{S}^{2}$, on a latitude circle $\mathrm{C}$ rotates an EM charge as pole $\mathrm{P}$, replacing the $g$ oscillation, at 0 a Higgs field sets the mass at rest for the EM lepton. In a Hopf blow up to the weak $\mathrm{S}^{3}$ sphere, points of $\mathrm{S}^{2}$ become fibers $\mathrm{S}^{1}$ (replacing U(1)), 0 is the core to which the EM torus for $\mathrm{C}$ can be retracted, the leaning circle for $\mathrm{P}$ is rotating about a central torus z-axis and spin has a 45 degree leaning angle $\theta$ towards the z-axis.

\section{Hilbert space octonians}

The eight dimensions of octonians have a complex meaning for a Hilbert space presentation $\mathrm{H}^{4}$ dimension as complex 4-dimensional space $\mathrm{C}^{4}$. The lattice $\mathrm{L}$ of (closed) subspaces consists as in the real case $\mathrm{R}^{4}$ of Boolean blocks $2^{4}$ where all subspaces and their

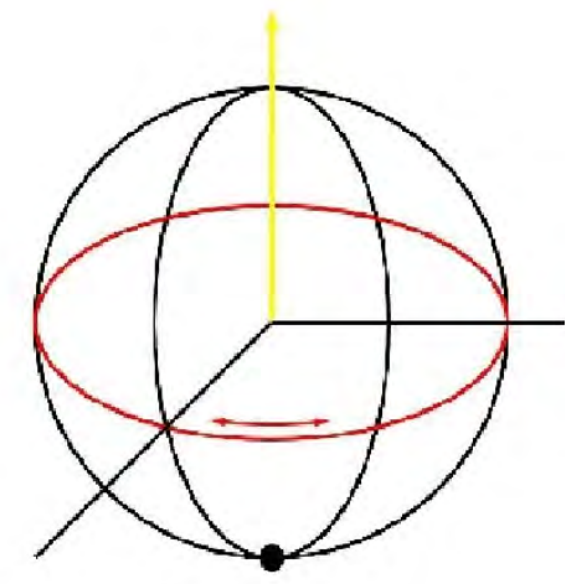

Figure 1: EM lepton $S^{2}$.
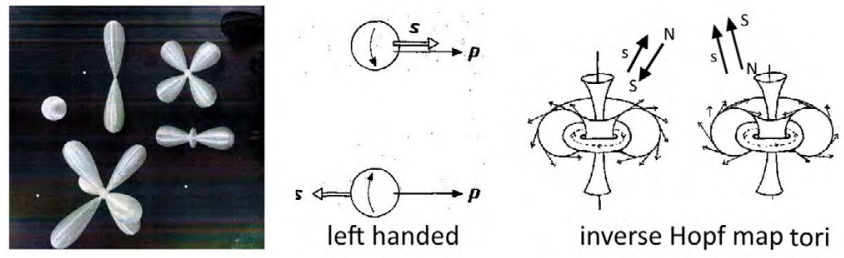

inverse Hopf map tori

Figure 3: Orbitals for electrons in atoms show their different Hopf locations as sphere, club, torus; at right the inverse Hopf map geometry of $\mathrm{S}^{2}$ in $\mathrm{S}^{3}$; in the middle the helicity of neutral leptons with spin $\mathrm{s}$ aligned with momentum $\mathrm{p}$.
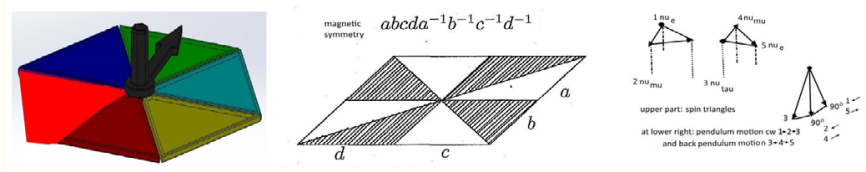

Figure 4: G-compass at left, magnetic symmetry (middle), pendulum oscillation at right. 
corresponding projection operators are commuting. Noncommuting means that $\mathrm{L}$ is the set theoretical union of its blocks. A block is drawn as an interval with four atoms marked for base vectors or lines in $\mathrm{H}^{4}$. For $\mathrm{H}=\mathrm{R}$ these are local coordinates spacetime $(\mathrm{x}, \mathrm{y}, \mathrm{z}, \mathrm{t})$, for $\mathrm{H}=\mathrm{C}$ octonian coordinates $\mathrm{e}_{\mathrm{j}}$ with $\mathrm{j}=1,2,3,4$ for these coordinates and complex with $\left(\mathrm{z}_{1}=\left(\mathrm{x}, \mathrm{e}_{5}\right), \mathrm{z}_{2}=\left(\mathrm{y}, \mathrm{e}_{7}\right), \mathrm{z}_{3}=\left(\mathrm{z}, \mathrm{e}_{0}\right), \mathrm{z}_{4}=\left(\mathrm{t}, \mathrm{e}_{6}\right)\right)$. This doubling of coordinates for the blocks in $\mathrm{C}^{4}$ has a meaning for keeping the lattice structure of $\mathrm{H}^{4}$ where the projections make the splitting of $\mathrm{H}^{4}$ in two orthogonal subspaces $\mathrm{U}+\mathrm{U}^{\perp}$, for $w \varepsilon \mathrm{C}$ as the complex presentation $\mathrm{w}=\mathrm{x}+$ iy. For a 4 -cycle (figure 5 at left) in $\mathrm{L}$ there must exist a central flash (Figure 5 at right).
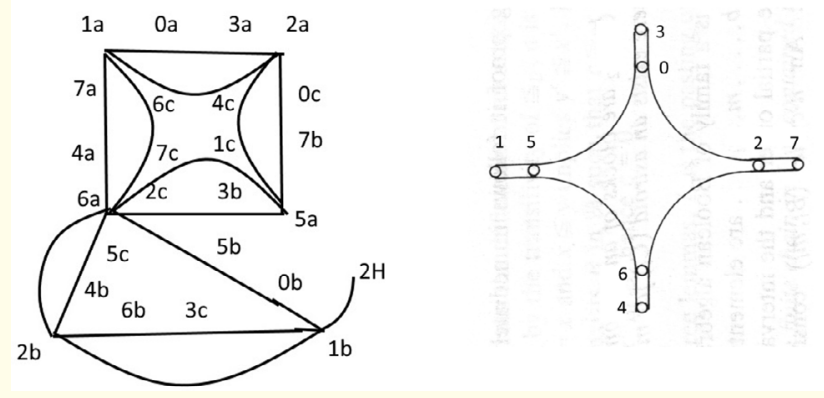

Figure 5: A sublattice diagram with a 4-cycle and a 3-cycle, flash with octonian coordinate indices; the lower triangle at left shows that in $\mathrm{L}$ this requires an additional block, containing its vertices.

In the flash two adjacent blocks have two atoms in common. The coupling is 15 for the Heisenberg uncertainty position-momentum $\left(\mathrm{x}, \mathrm{e}_{5}\right)$ and 46 time energy $\left(\mathrm{t}, \mathrm{e}_{6}\right)$. The Heisenberg uncertainty angle angular momentum is not presented. It is replaced by $(2,7)$ and $(0,3)$. The last case gives a new EM-compass uncertainty with maximal bound speed of light $\mathrm{c}$ for universes matter speeds [1-11].

In case an uncertainty for $(2,7)$ is wanted, the mass rescalings for matter systems can be quoted.

As measuring GF it is $(2,5,7)$. Mass can be renormed through Minkowski metrical computations through relative speed between two coordinate systems (using a $\sin \varphi=\mathrm{v} / \mathrm{c}$ rescaling), additive rescaled by measuring inner frequencies for speeds/energies in the system through $\mathrm{mc}^{2}=\mathrm{hf}$ or observed in mass defects. The co- ordinate 7 is then a $\psi$ function for it and is observable through a probability distribution $\psi c(\psi)$. As a wave package for a system, the Minkowski rescaling gives a common (optical computed) group speed with which it moves in the environment. The former neutrino oscillation is another form of observing their different masses. A Higgs field uses different approaches for attributing mass to a system and for setting barycenters of combined systems where a newly computed mass is set. The weak decay allows that an intermediate barycenter of a weak boson is annihilated and two generated systems from it get two (or no) different barycenters. For two systems, when a gravitational interaction is generated for them, a common barycenter is set and used by Higgs for attributing mass to the new system. The Schwarzschild radius Rs of a system is used when it belongs to a huge system Q (as sun) and an orbiting system $P$ (as planet). It sets for rescaling time as differential in the tangent space a dt rescaling angle $\sin \beta=\mathrm{Rs} / \mathrm{r}$ as $\mathrm{dt}^{\prime}=\mathrm{dt} \cdot \cos \beta$.

In both cases, Schwarzschild and Minkowski the area between a space and time coordinate is preserved, $\mathrm{dr}^{\prime} \mathrm{dt}^{\prime}=\mathrm{dr} \cdot \mathrm{dt}, \mathrm{l}^{\prime} \mathrm{t}^{\prime}=\mathrm{l} \cdot \mathrm{t}$. For $\mathrm{dr}$ the rescaling is then $\mathrm{dr}^{\prime}=\mathrm{dr} /(\cos \beta)$. Both rescalings use orthogonal projections beween two rays in an angle $\beta$ or $\varphi$ (Figure 6). For $\beta$ the mathematical inversion of a mass system at the Schwarzschild radius of a black hole inverts its radius $r$ in $r^{4} r=R^{2}$ when absorbed. The angle $\varphi$ speed $v$ is inverted for dark energy systems in $v^{\prime} v=c^{2}$. At the borders, the electromagnetic waves have speed $v=c$ and Higgs bosons in fields may use $r=$ Rs. The normed Schwarzschild radius matrix is $G$ of order 6 from the G-compass, having first row ( $1-1)$ and second row (1 0$)$. B of order 8 for the EM-compass has the second row replaced by $(11)$.

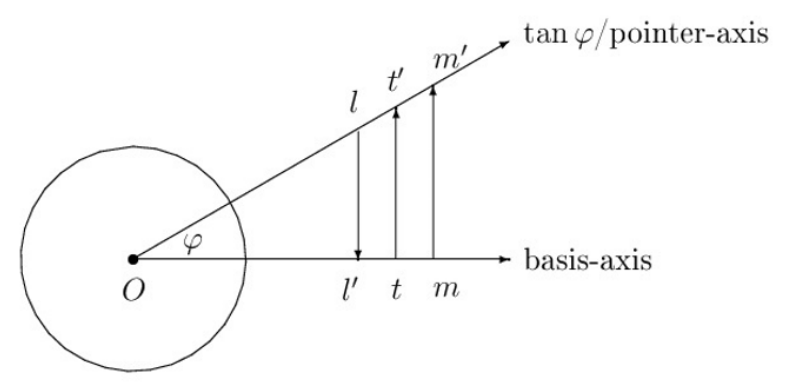

Figure 6: Minkowski watch for rescaling of length, time, mass as orthogonal, spiralic projection. 
The Minkowski scaled matrix M of order 2 has the columns of $\mathrm{G}$ interchanged, as Moebius transformation it is $(-\mathrm{v}+\mathrm{c}) / \mathrm{c}, \mathrm{G}$ is ( $\mathrm{r}-$ $\mathrm{Rs}) / \mathrm{r}$ and for $\mathrm{B}$ the scaling factor is $1 / \sqrt{2}$ as proportion between the first and second cosmic speed of a matter system. $\mathrm{B}^{2}$ is the real Pauli matrix $\sigma_{2}$ with first row $\left(\begin{array}{ll}0 & 1\end{array}\right)$ and second row $(-10)$. In case a two ray angle is wanted for $B$, it is the $i^{1 / 2} 45$ degree angle, for $G$ it is replaced by 60 degree and for $m 180$ degree. The $8^{\text {th }}, 6^{\text {th }}$ or square roots belong to characteristic polynomials $z^{n}-1, n=8,6,2$. Difference equations replace differential equations and allow 8,6, or 2 solutions. For $\mathrm{M}$ the speed $\mathrm{v}$ can be signed to $+\mathrm{v}$ or $-\mathrm{v}$ for Lorentz transformations.

All matrices use as rows different bases in a plane E. $\mathrm{B}^{2}$ is for presenting complex numbers $\mathrm{z}=\mathrm{x}+$ iy in matrix form $\mathrm{z}=\mathrm{x} \cdot \mathrm{id}+\mathrm{y} \cdot \sigma_{2}$ with first row ( $\mathrm{x} y$ ) and second row (-y $\mathrm{x})$. The quaternionic extension $\mathrm{Q}$ of $\mathrm{C}$ uses for $\sigma_{1}, \sigma_{3}$ other bases of $\mathrm{E}$. Euler angles generate the quaternions noncommutative Pauli matrices.

As a research project is posed: look at the operator or matrix transformations between the different $2 \times 2$-matrices in use. Included in the study can be the 3 x 3-GellMann matrices of SU(3), extended from Pauli matrices. In complex form, the above matrix for complex numbers as $2 \times 2$-matrix is in quaternions the matrix with complex numbers $z_{1}=z+i c t, z_{2}=x+i y$, in the first row $\left(z_{1} z_{2}\right)$, in the second row $\left(-c\left(z_{2}\right) c\left(z_{2}\right)\right)$. In $S U(3)$ matrices $z_{3}=(m, f)=\left(e_{5}, e_{6}\right) i$ added as cross product $\mathrm{z}_{3}=\mathrm{z}_{1} \mathrm{xz}_{2}$ and the three 4-dimensional spaces are in matrix form written, having a last row and column with 0 coordinates added or inserting them in the second or first position. Their first rows are then $\left(\mathrm{z}_{1} \mathrm{z}_{2} 0\right),\left(\mathrm{z}_{1} 0 \mathrm{z}_{3}\right),\left(0 \mathrm{z}_{2} \mathrm{z}_{3}\right)$. The octonian cross product $z_{4}=z_{1} x_{2} x_{3}$ is missing. The extension from $S U(2)$ to SU(3) allows another multiplication table than the octonians and other measuring triples GF.

\section{Bibliography}

1. G Kalmbach HE. "MINT-WIGRIS". MINT Verlag, Bad Woerishofen (2017).

2. G Kalmbach HE. "(Chef-Hrsg), MINT (Mathematik, Informatik, Naturwissenschaften, Technik)". MINT Verlag, Bad Woerishofen, volume 1-65 (1997-2020).

3. Internet video under YouTube: Moebius Transformations Revealed (2014).
4. G Kalmbach and U Eberspaecher. "MINT-Wigris Tool Bag". Bad Woerishofen (2019-2021).

5. G Kalmbach. "MINT-Wigris Postulates". In: researchgate.net under MINT-Wigris Project (2020).

6. E Schmutzer. "Projektive einheitliche Feldtheorie". Harry Deutsch, Frankfurt (2004).

7. K Stierstadt. "Physik der Materie”. VCH, Weinheim (1989).

8. G Kalmbach HE. MINT-Wigris project. In the internet under: researchgate.net.

9. Kalmbach G. "Orthomodular Lattices". London New York: Academic Press (1983): 390.

10. T Poston and I Stewart. "Catastrophe theory and its applications". Pitman, London (1978).

11. G Kalmbach HE. "A Heisenberg c-compass". Journal of Applied Material Science and Engineering Research 4.2 (2020): 63-67.

\section{Assets from publication with us}

- Prompt Acknowledgement after receiving the article

- Thorough Double blinded peer review

- Rapid Publication

- Issue of Publication Certificate

- High visibility of your Published work

Website: www.actascientific.com/

Submit Article: www.actascientific.com/submission.php Email us: editor@actascientific.com

Contact us: +919182824667 